\title{
Extended Nonsingular Terminal Sliding Surface for Second-Order Nonlinear Systems
}

\author{
Ji Wung Jeong, Ho Suk Yeon, and Kang-Bak Park \\ Department of Control and Instrumentation Engineering, Korea University, 2511 Sejong-ro, Sejong City 339-700, Republic of Korea \\ Correspondence should be addressed to Kang-Bak Park; kbpark@korea.ac.kr
}

Received 29 January 2014; Accepted 18 April 2014; Published 11 May 2014

Academic Editor: Hui Zhang

Copyright (C) 2014 Ji Wung Jeong et al. This is an open access article distributed under the Creative Commons Attribution License, which permits unrestricted use, distribution, and reproduction in any medium, provided the original work is properly cited.

\begin{abstract}
An extended nonsingular terminal sliding surface is proposed for second-order nonlinear systems. It is shown that the proposed surface is a superset of a conventional nonsingular terminal sliding surface which guarantees that the system state gets to zero in finite time. The conventional nonsingular sliding surfaces have been designed using a power function whose exponent is a rational number with positive odd numerator and denominator. The proposed nonsingular terminal sliding surface overcomes the restriction on the exponent of a power function; that is, the exponent can be a positive real number. Simulation results are provided to show the validity of the main result.
\end{abstract}

\section{Introduction}

According to the progress of control schemes, a variety of nonlinear control systems have been proposed: H-infinity optimal control, fuzzy control, neural network control, controller using genetic algorithms, and so on [1-4]. Sliding mode control (SMC) method, which is also called a variable structure system (VSS), is one of nonlinear robust control schemes. It has been widely used because of its invariance properties to parametric uncertainties and external disturbances [5-8]. In conventional sliding mode control systems, sliding surfaces have been designed such that the overall system in the sliding mode is asymptotically stable. However, the asymptotic stability does not guarantee a finite time convergence.

Although most of control systems proposed so far have been designed such that the closed-loop system is asymptotic, finite time stabilization is very important to many actual applications, such as motor, power, robot, and aerospace systems because, in the actual applications, the main objective of a control system is to make system's state to a desired one in a finite time interval which is determined a priori. Thus, many recent studies have focused on the finite time stabilization [9$13]$.
Finite time control systems based on sliding mode control schemes have been called terminal sliding mode control systems since their sliding surfaces have been designed as terminal attractors [14]. Recently, terminal sliding mode control systems have been studied to achieve a finite time convergence in many applications [15-17]. Conventional terminal sliding mode controllers used terminal sliding surfaces which were designed using a power function of a system state. They guaranteed that the system state reached zero in finite time. On the contrary, however, they suffered from singularity problems and had restrictions on the range of the exponent of a power function. The exponent should be a rational number with positive odd numerator and denominator [18]. In order to avoid the singularity problem in conventional terminal sliding mode control systems, nonsingular terminal sliding surfaces have been proposed very recently for robot manipulators $[19,20]$. However, the same restriction on the exponent of a power function in the nonsingular sliding surface still remained: the exponent should be a rational number with a positive odd numerator and denominator.

Thus, in this paper, a novel nonsingular terminal sliding surface is proposed for second-order nonlinear systems. It is shown that the proposed scheme guarantees that the system state gets to zero in finite time and it does not suffer from 
singularity problems. Furthermore, the proposed nonsingular terminal sliding surface overcomes the restriction on the exponent of a power function by being a superset of a conventional nonsingular terminal sliding surface. That is, we extend the range of an exponent of a power function in the nonsingular terminal sliding surface from a rational number with an odd numerator and an odd denominator to a real number.

Simulation results and experimental results are given to show the validity of the main result.

\section{Main Results}

Consider a second-order nonlinear system of the following form:

$$
\ddot{x}=f(x, \dot{x}, t)+g(x, \dot{x}, t)(u+d(x, \dot{x}, t)),
$$

where $x$ and $\dot{x}$ are state variables, $f(x, \dot{x}, t)$ is a nonlinear term, $u$ is a scalar input, $g(x, \dot{x}, t) \neq 0 \forall x, \dot{x} \in R, \forall t \in$ $R_{+}$, and $d(x, \dot{x}, t)$ represents the uncertainties and external disturbances. It is assumed that the following assumption holds.

Assumption 1. The uncertainty $d(x, \dot{x}, t)$ is bounded as follows:

$$
|d(x, \dot{x}, t)| \leq D(x, \dot{x}, t), \quad \forall x, \dot{x} \in R, \forall t \in R_{+},
$$

where $D(x, \dot{x}, t)$ is a known positive function.

In previous works on terminal sliding mode control systems, the conventional terminal sliding surfaces have been designed as

$$
s_{1}=\dot{x}+c_{1} x^{q_{1} / r_{1}},
$$

where $c_{1}>0,0<q_{1} / r_{1}<1$, and $q_{1}$ and $r_{1}$ are positive odd integers [8-10]. However, though the conventional terminal sliding surface in (3) ensures finite time convergence, it suffers from the singularity problem and has a restriction on the exponent of the power function [14]. In the phase space with $x$ and $\dot{x}$ axes, the set of singular points is a vertical axis except for at the origin; that is,

$$
S=\{(x, \dot{x}) \mid x=0, \dot{x} \neq 0\} .
$$

Recently, a nonsingular terminal sliding surface was proposed to overcome the singularity problem [12]

$$
s_{2}=\dot{x}^{q_{2} / r_{2}}+c_{2} x,
$$

where $c_{2}>0,1<q_{2} / r_{2}<2$, and $q_{2}, r_{2}$ are positive odd integers

However, this surface still has the restrictions on the exponent of the power function; that is, $q_{2}, r_{2}$ should be positive odd integers. Thus, we propose an extended nonsingular terminal sliding surface whose exponent can be any real number.

$$
s=|\dot{x}|^{1 / p} \operatorname{sgn}(\dot{x})+c \cdot x,
$$

where $\operatorname{sgn}(\cdot)$ is the signum function, $c$ is a positive constant, and $1 / 2 \leq p<1$ is a real number.

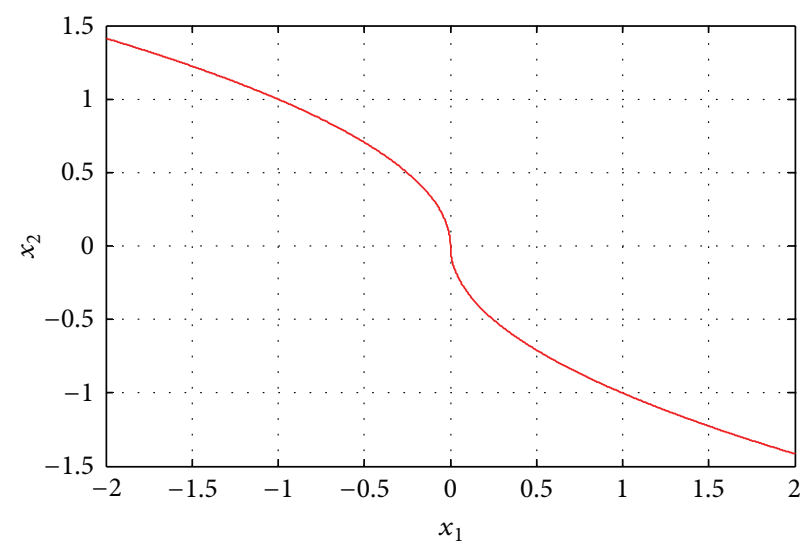

FIGURE 1: Example of a nonsingular terminal sliding surface.

Figure 1 shows a typical nonsingular terminal sliding surface. Clearly, for any $c>0$ and $1 / 2 \leq p<1$, the phase portrait is in the second and fourth quadrants in the phase space with the axes of $x_{1}=x$ and $x_{2}=\dot{x}$.

Remark 2. It is clear that the proposed nonsingular terminal sliding surface is stable because the sliding surface is in the second and fourth quadrants in the phase space with the axes of $x$ and $\dot{x}$, where this property implies that $x \cdot \dot{x}<0$ for all $x \neq 0$.

For the proposed nonsingular terminal sliding surface, we derived the following theorem for the finite time convergence.

Theorem 3. The proposed nonsingular terminal sliding surface (6) guarantees that the system state gets to zero in finite time in the sliding mode, $s=0$, and the relaxation time [18] is

$$
t_{r}=\frac{|x(0)|^{1-p}}{c^{p}(1-p)} .
$$

Proof. If the system is in the sliding mode and $\dot{x} \geq 0$, the proposed sliding surface (6) can be rewritten as follows:

$$
\dot{x}^{(1 / p)}+c x=0
$$

From the above equation, if $\dot{x}(0) \geq 0$, that is, $x(0) \leq 0$, the following equations can be easily derived:

$$
\begin{aligned}
& \dot{x}^{(1 / p)}+c x=0 \\
& \Longleftrightarrow \frac{d x}{d t}=c^{p}(-x)^{p} \\
& \Longleftrightarrow \frac{d x}{(-x)^{p}}=c^{p} d t \\
& \Longleftrightarrow-\frac{d(-x)}{(-x)^{p}}=c^{p} d t \\
& \Longleftrightarrow-\int_{-x=-x(0)}^{-x=0} \frac{d(-x)}{(-x)^{p}}=\int_{0}^{t_{r}} c^{p} d t
\end{aligned}
$$




$$
\begin{aligned}
& \left.\Longleftrightarrow \frac{(-x)^{(1-p)}}{1-p}\right|_{-x=-x(0)} ^{-x=0}=c^{p} t_{r} \\
& \Longleftrightarrow t_{r}=\frac{(-x(0))^{(1-p)}}{c^{p}(1-p)}=\frac{|x(0)|^{(1-p)}}{c^{p}(1-p)},
\end{aligned}
$$

where $t_{r}$ represents the relaxation time [18].

If $\dot{x}(0)<0$, that is, $x(0)>0, t_{r}$ can be derived in a similar way:

$$
\begin{aligned}
&-(-\dot{x})^{(1 / p)}+c x=0 \\
& \Longleftrightarrow-\dot{x}=c^{p} x^{p} \\
& \Longleftrightarrow-\frac{d x}{d t}=c^{p} x^{p} \\
& \Longleftrightarrow-\frac{d x}{x^{p}}=c^{p} d t \\
& \Longleftrightarrow-\int_{x(0)}^{0} x^{-p} d x=\int_{0}^{t_{r}} c^{p} d t \\
& \Longleftrightarrow-\left.\frac{x^{(1-p)}}{1-p}\right|_{x=x(0)} ^{x=0}=c^{p} t_{r} \\
& \Longleftrightarrow t_{r}=\frac{x(0)^{(1-p)}}{c^{p}(1-p)}=\frac{|x(0)|^{(1-p)}}{c^{p}(1-p)} .
\end{aligned}
$$

This completes the proof.

In the above theorem, we proved that the system state gets to zero in finite time if it is in the sliding mode. Thus, in the following theorem, we propose a controller that guarantees that the sliding mode existence condition holds such that the overall system will be in the sliding mode.

Theorem 4. For system (1) with the proposed nonsingular terminal sliding surface (6), the following controller guarantees that the sliding mode existence condition holds:

$$
\begin{aligned}
u= & \frac{1}{g(x, \dot{x})} \\
& \times\left(-f(x, \dot{x})-c p|\dot{x}|^{(2-(1 / p))} \operatorname{sgn}(\dot{x})-k_{1} s-k_{2} \operatorname{sgn}(s)\right),
\end{aligned}
$$

where $k_{1}>0, k_{2}>|g|(D+\alpha), \alpha$ is a positive constant and $1 / 2 \leq p<1$.

Proof. Let the Lyapunov function candidate be

$$
V(s)=\frac{1}{2} s^{2} .
$$

Applying (1) and (6) to $d V / d t=\dot{V}$, the following equations can be obtained if $\dot{x}>0$; that is, $x<0$ :

$$
\begin{aligned}
\dot{V}(s) & =s \dot{s}=s\left(\frac{1}{p} \dot{x}^{((1 / p)-1)} \ddot{x}+c \dot{x}\right) \\
& =s\left(\frac{1}{p} \dot{x}^{((1 / p)-1)}(f+g(u+d))+c \dot{x}\right)
\end{aligned}
$$

$$
\begin{aligned}
& \leq s\left(\frac{1}{p} \dot{x}^{((1 / p)-1)}\right)\left(-k_{1} s-\alpha \operatorname{sgn}(s)\right) \\
& \leq \frac{1}{p} \dot{x}^{((1 / p)-1)}\left(-k_{1} s^{2}-\alpha|s|\right) .
\end{aligned}
$$

Similarly, if $\dot{x}<0$, that is, $x>0$, the following equations can be derived:

$$
\begin{aligned}
\dot{V}(s) & =s \dot{s}=s\left(\frac{1}{p}(-\dot{x})^{((1 / p)-1)}(-\ddot{x})(-1)+c \dot{x}\right) \\
& =s\left(\frac{1}{p}(-\dot{x})^{((1 / p)-1)}(f+g(u+d))+c \dot{x}\right) \\
& \leq s\left(\frac{1}{p} \dot{x}^{((1 / p)-1)}\right)\left(-k_{1} s-\alpha \operatorname{sgn}(s)\right) \\
& \leq \frac{1}{p}(-\dot{x})^{((1 / p)-1)}\left(-k_{1} s^{2}-\alpha|s|\right) .
\end{aligned}
$$

Equations (13) and (14) can be represented as

$$
\dot{V}(s)=-\frac{1}{p}|\dot{x}|^{((1 / p)-1)}\left(k_{1} s^{2}+\alpha|s|\right) .
$$

Here, $\dot{V}(s)<0$ if $|\dot{x}| \neq 0$ and $s \neq 0$.

If $|\dot{x}|=0$, from (1) and (11), the following equation can be obtained:

$$
\ddot{x}=\frac{d \dot{x}}{d t}=-k_{1} s-k_{2} \operatorname{sgn}(s)+g \cdot d
$$

which implies that

$$
\left.s \frac{d \dot{x}}{d t}\right|_{\dot{x}=0} \leq-k_{1} s^{2}-\alpha|s| .
$$

Thus, it is clear that if $|\dot{x}|=0$ and $s \neq 0, \dot{x}$ deviates from zero; that is, the set of points, $|\dot{x}|=0$ and $s \neq 0$, is not an attractor. In phase space, these points are all points on the horizontal axis that save the origin.

Therefore, we can conclude that $s$ goes to zero.

Remark 5. Since the proposed controller (11) has a term $|\dot{x}|^{(2-(1 / p))}$, it is clear that in order to avoid the singularity problem one should choose the control parameter, $p$, such that $1 / 2 \leq p<1$, even though the finite time convergence can be obtained for $0<p<1$ in (6).

\section{Examples}

To show the validity of the proposed method, the simulation results for the following system are given:

$$
\ddot{x}=20 \dot{x}|\dot{x}|+10 \sin (5 t) \sqrt{|x|}+10(u+d),
$$

where $d(x, \dot{x}, t)=2 \sqrt{x}+\sin (10 t)$. The control parameters were chosen as follows: $c=5, k_{1}=2, k_{2}=10(2 \sqrt{|x|}+1+$ $\alpha$ ), and $\alpha=1$. When the initial condition is $x(0)=1$, from 


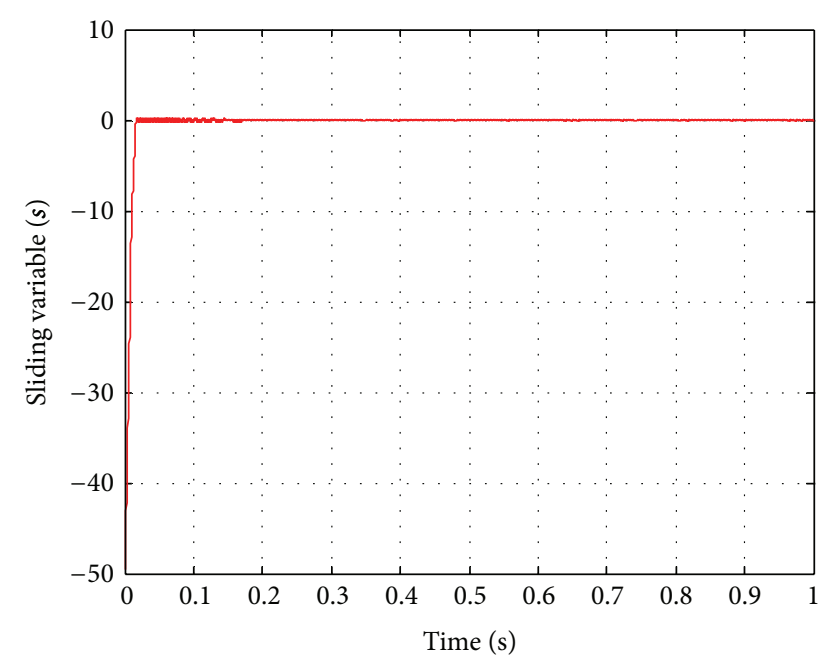

FIGURE 2: Sliding variable $(s)$.

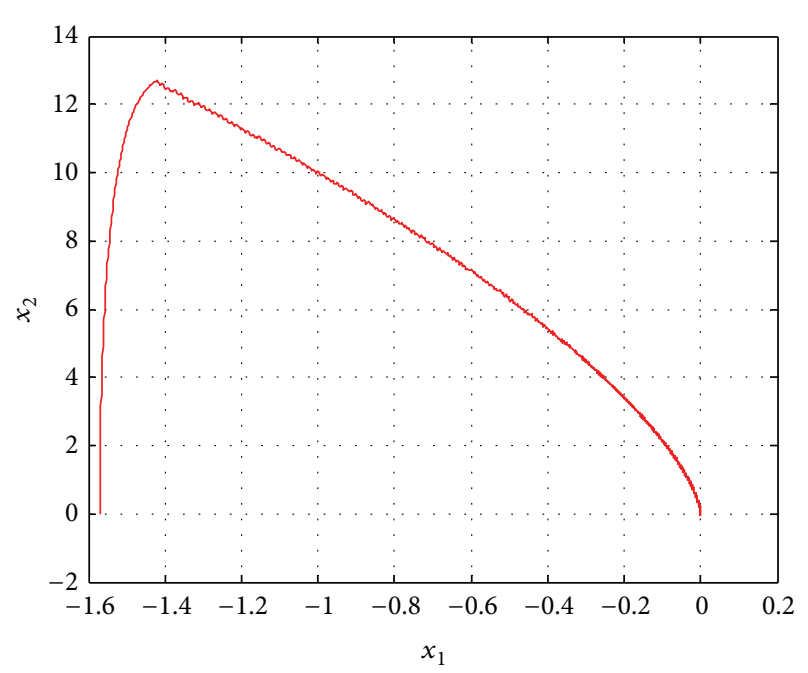

Figure 3: Phase portrait ( $x$ versus $\dot{x})$.

(18), $p=1 / 2$ is obtained for the minimum relaxation time. Clearly, $p=1 / 2$ is out of a conventional nonsingular terminal sliding surface. In the following figures, $x_{1}, x_{2}$ represent $x, \dot{x}$, respectively.

Figures $2-5$ show the simulation results. Figure 2 represents the profile of the sliding variable, $s$. It is shown that the system state stayed on the nonsingular terminal sliding surface all the time after it hit the sliding surface for the first time.

It is clear that the sliding mode existence condition, $s \cdot \dot{s}<$ 0 , was satisfied all the time and it was also known from the phase portrait given in Figure 3.

Figure 4 shows that the output zeroed in finite time.

The control input signal can be seen in Figure 5.

To show the nonsingular property, we simulated the system with the initial condition near the vertical axis in the phase space because, from (4), a set of singular points for the conventional terminal sliding surface is a vertical

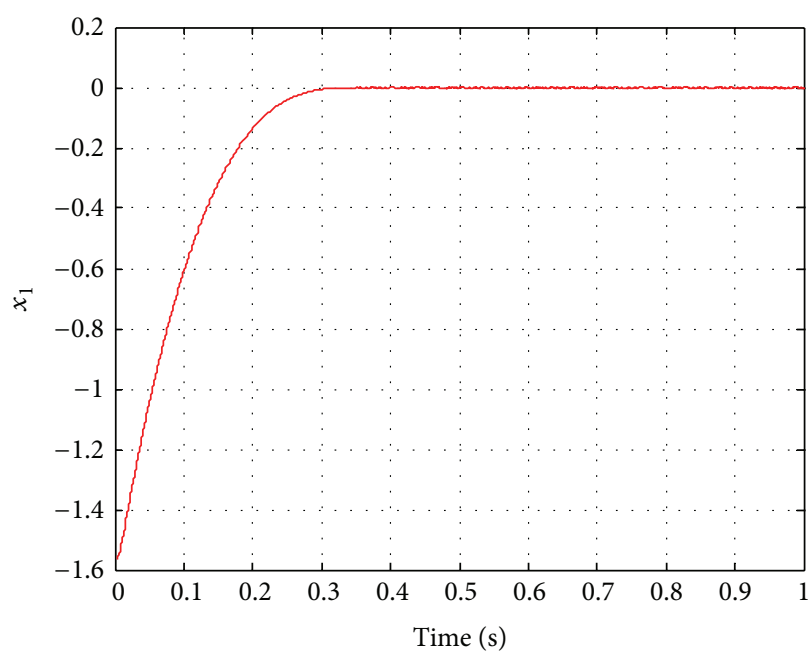

FIgURE 4: Output $(x)$.

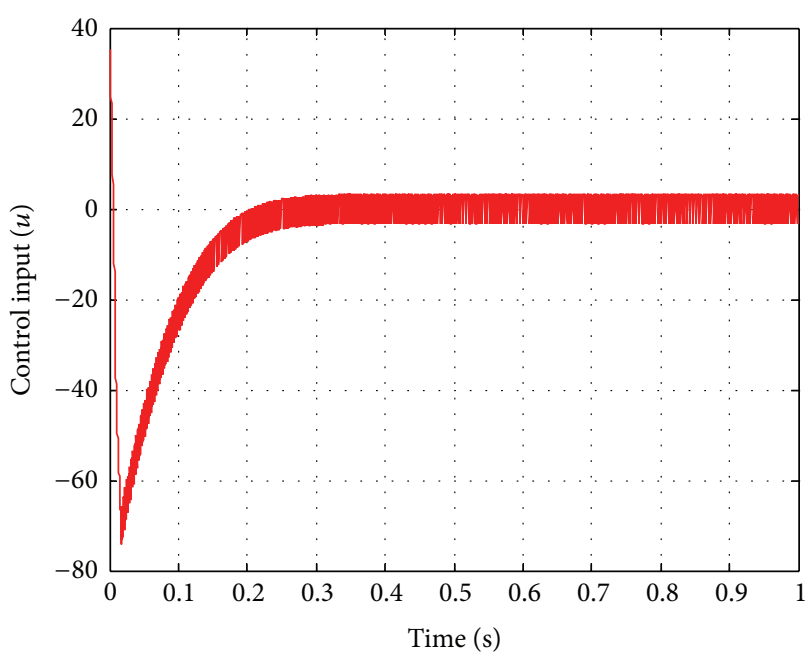

Figure 5: Control input $(u)$ for signum function.

axis in the phase space. Figures 6 and 7 show the results. Figure 6 represents that the system state zeroed even though it crossed over the vertical axis, a set of singular points for a conventional terminal sliding surface. In the proof of Theorem 4, we showed that the horizontal axis is not an attractor so that the system state on this axis moves out of the axis. This is also verified from Figure 6, since the system state also crossed the horizontal axis. The control input is shown in Figure 7. It can be seen that it did not suffer from the singularity problem.

In addition, we also applied the proposed method to the actual DC motor system. For the control unit in the experimental system, a TMS320F2812 DSP processor was used. The sampling time was set to $1 \mathrm{msec}$. The following model was used for the DC motor system:

$$
\ddot{x}=-40.65 \dot{x}+46.67 u \text {. }
$$

Figure 8 shows that the motor position converges zero in finite time. 


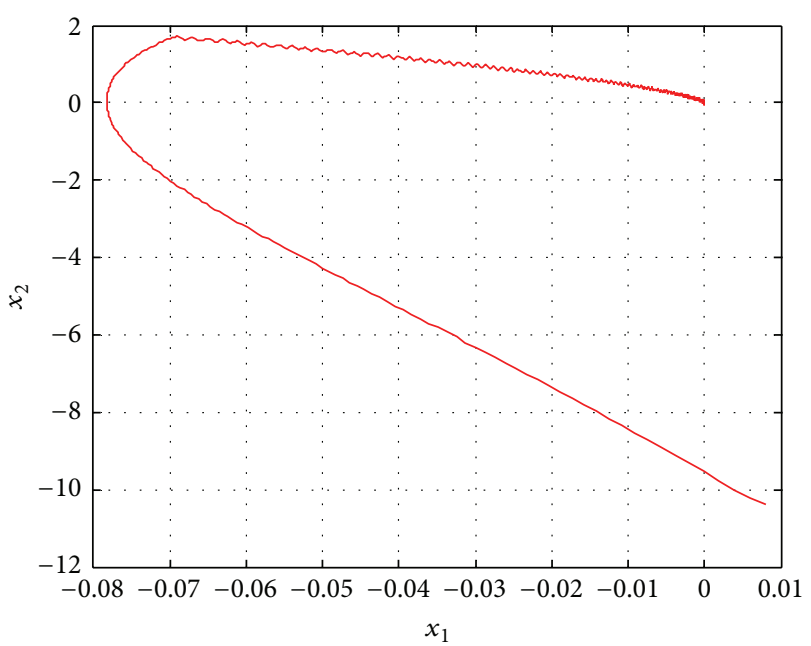

FIGURE 6: Phase portrait ( $x$ versus $\dot{x}$ ).

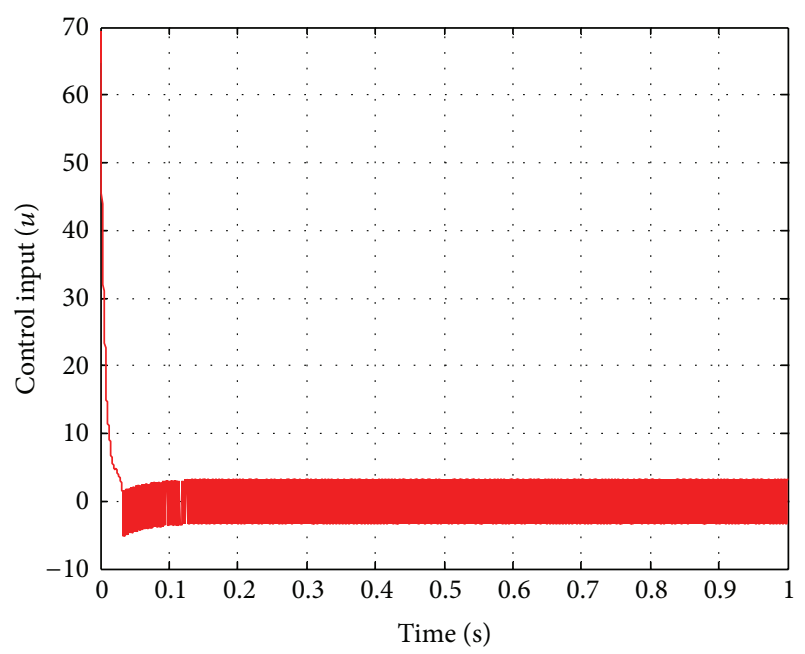

FIgURE 7: Control input $(u)$.

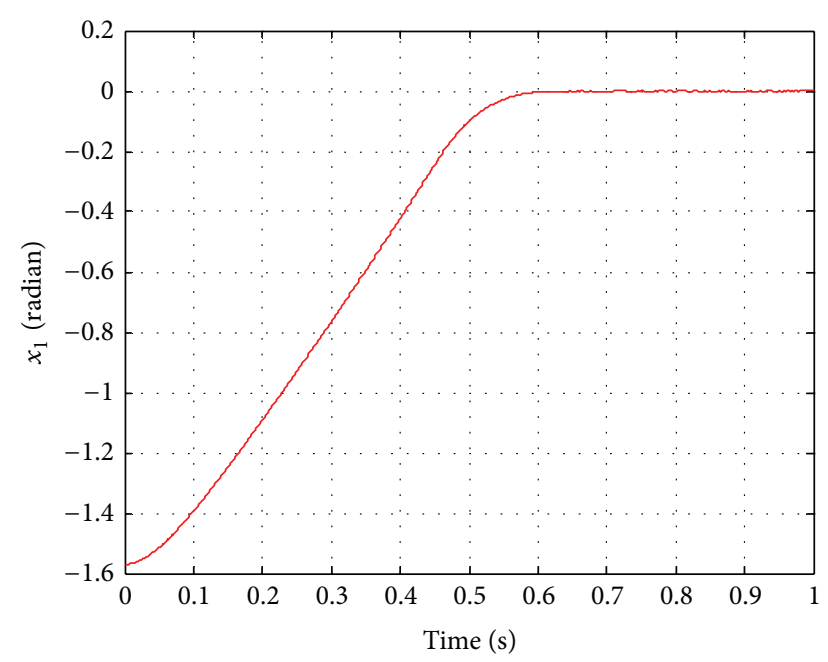

FIgURE 8: Output angle $(x)$.

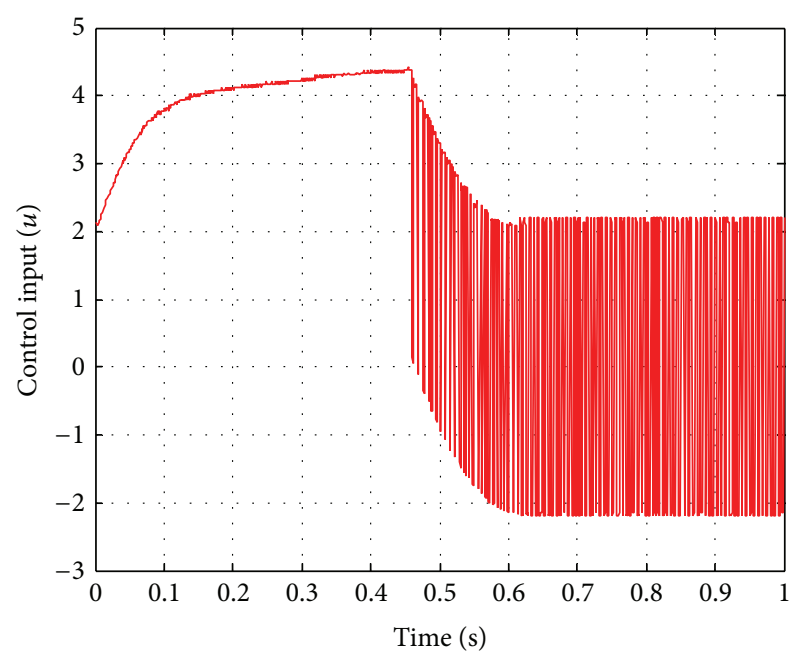

Figure 9: Control input $(u)$.

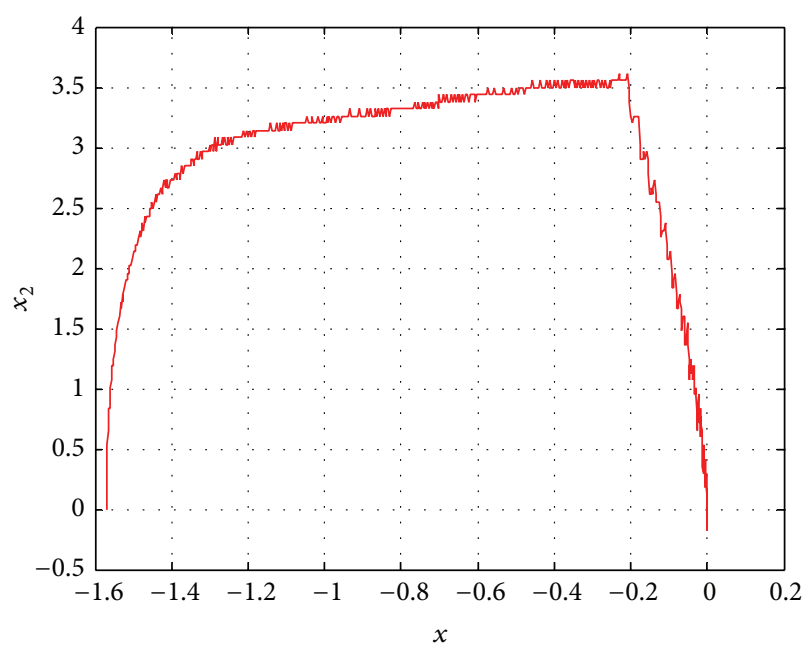

Figure 10: Phase portrait ( $x$ versus $\dot{x})$.

The control input signal is given in Figure 9. It represents that the control signal is bounded all the time.

Figure 10 shows a phase portrait. It is clear that the sliding mode existence condition, $s \cdot \dot{s}<0$, was satisfied all the time.

\section{Conclusions}

In this paper, the extended nonsingular terminal sliding surface for second-order nonlinear systems has been proposed. It has been shown that the proposed nonsingular terminal sliding surface guarantees finite time convergence and is singularity-free. Furthermore, the exponent of the power function in the proposed sliding surface can be a real number in contrast to conventional nonsingular terminal sliding surfaces where the exponent should be a rational number with an odd numerator and odd denominator. Simulation and experimental results have shown the validity of the main result. 


\section{Conflict of Interests}

The authors declare that there is no conflict of interests regarding the publication of this paper.

\section{Acknowledgment}

This work was supported by a Korea University Grant.

\section{References}

[1] T. Başar and P. Bernhard, $H^{\infty}$ Optimal Control and Related Minimax Design Problems: A Dynamic Game Approach, 2008.

[2] J. H. Lilly, Fuzzy Control and Identification, 2010.

[3] J. Sarangapani, Neural Network Control of Nonlinear DiscreteTime Systems, 2006.

[4] K. F. Man, K. S. Tang, and W. A. Halang, Genetic Algorithms for Control and Signal Processing, 2011.

[5] J. Y. Hung, W. Gao, and J. C. Hung, "Variable structure control. A survey," IEEE Transactions on Industrial Electronics, vol. 40, no. 1, pp. 2-22, 1993.

[6] K. D. Young, V. I. Utkin, and Ü. Özgüner, "A control engineer's guide to sliding mode control," IEEE Transactions on Control Systems Technology, vol. 7, no. 3, pp. 328-342, 1999.

[7] N. Hung, J. S. Im, S.-K. Jeong, H.-K. Kim, and S. B. Kim, "Design of a sliding mode controller for an automatic guided vehicle and its implementation," International Journal of Control, Automation and Systems, vol. 8, no. 1, pp. 81-90, 2010.

[8] B.-H. Lee, J.-H. Choo, S. Na, P. Marzocca, and L. Librescu, "Sliding mode robust control of supersonic three degrees-offreedom airfoils," International Journal of Control, Automation and Systems, vol. 8, no. 2, pp. 279-288, 2010.

[9] Y. Wu, B. Wang, and G. D. Zong, "Finite-time tracking controller design for nonholonomic systems with extended chained form," IEEE Transactions on Circuits and Systems II: Express Briefs, vol. 52, no. 11, pp. 798-802, 2005.

[10] Y. Hong and Z.-P. Jiang, "Finite-time stabilization of nonlinear systems with parametric and dynamic uncertainties," IEEE Transactions on Automatic Control, vol. 51, no. 12, pp. 1950-1956, 2006.

[11] G. Garcia, S. Tarbouriech, and J. Bernussou, "Finite-time stabilization of linear time-varying continuous systems," IEEE Transactions on Automatic Control, vol. 54, no. 2, pp. 364-369, 2009.

[12] C. B. Njima, W. B. Mabrouk, H. Messaoud, and G. Garcia, "Finite time stabilization of the four tanks system: extensions to the uncertain systems," Abstract and Applied Analysis, vol. 2014, Article ID 964143, 7 pages, 2014.

[13] Y. Yang and G. Chen, "Finite time stability of stochastic hybrid systems," Abstract and Applied Analysis, vol. 2014, Article ID 867189, 7 pages, 2014.

[14] S. Janardhanan and B. Bandyopadhyay, "On discretization of continuous-time terminal sliding mode," IEEE Transactions on Automatic Control, vol. 51, no. 9, pp. 1532-1536, 2006.

[15] L. Wang, T. Chai, and L. Zhai, "Neural-network-based terminal sliding-mode control of robotic manipulators including actuator dynamics," IEEE Transactions on Industrial Electronics, vol. 56, no. 9, pp. 3296-3304, 2009.

[16] L. Hui and J. Li, “Terminal sliding mode control for spacecraft formation flying," IEEE Transactions on Aerospace and Electronic Systems, vol. 45, no. 3, pp. 835-846, 2009.
[17] J. Li and L. Yang, "Finite-time terminal sliding mode tracking control for piezoelectric actuators," Abstract and Applied Analysis, vol. 2014, Article ID 760937, 9 pages, 2014.

[18] K.-B. Park and J.-J. Lee, "Comments on: 'a robust MIMO terminal sliding mode control scheme for rigid robotic manipulators"' IEEE Transactions on Automatic Control, vol. 41, no. 5, pp. 761-762, 1996.

[19] Y. Feng, X. Yu, and Z. Man, "Non-singular terminal sliding mode control and its application for robot manipulators," in Proceedings of the IEEE International Symposium on Circuits and Systems (ISCAS '01), pp. 545-548, May 2001.

[20] M. Jin, J. Lee, P. H. Chang, and C. Choi, "Practical nonsingular terminal sliding-mode control of robot manipulators for highaccuracy tracking control," IEEE Transactions on Industrial Electronics, vol. 56, no. 9, pp. 3593-3601, 2009. 


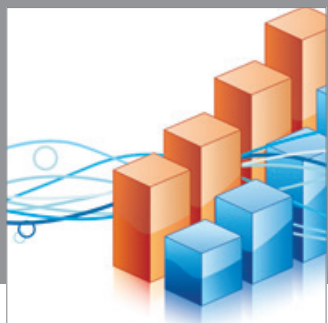

Advances in

Operations Research

mansans

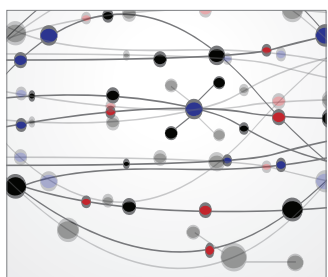

The Scientific World Journal
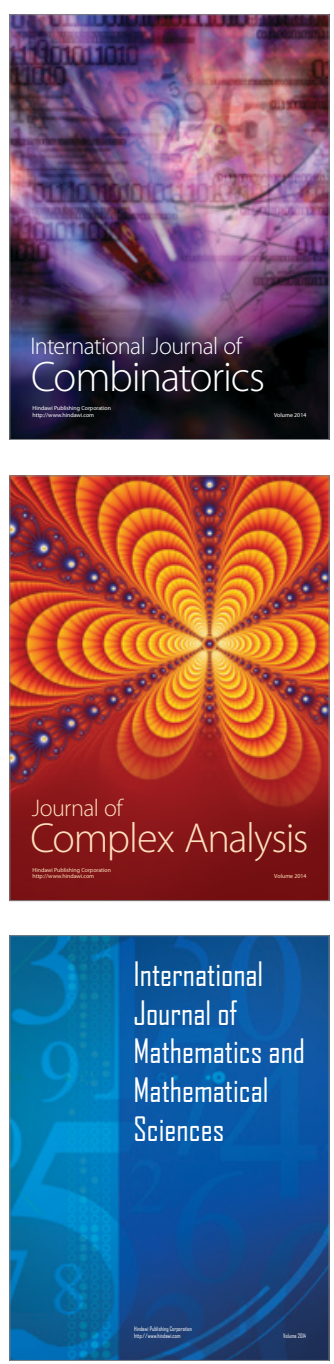
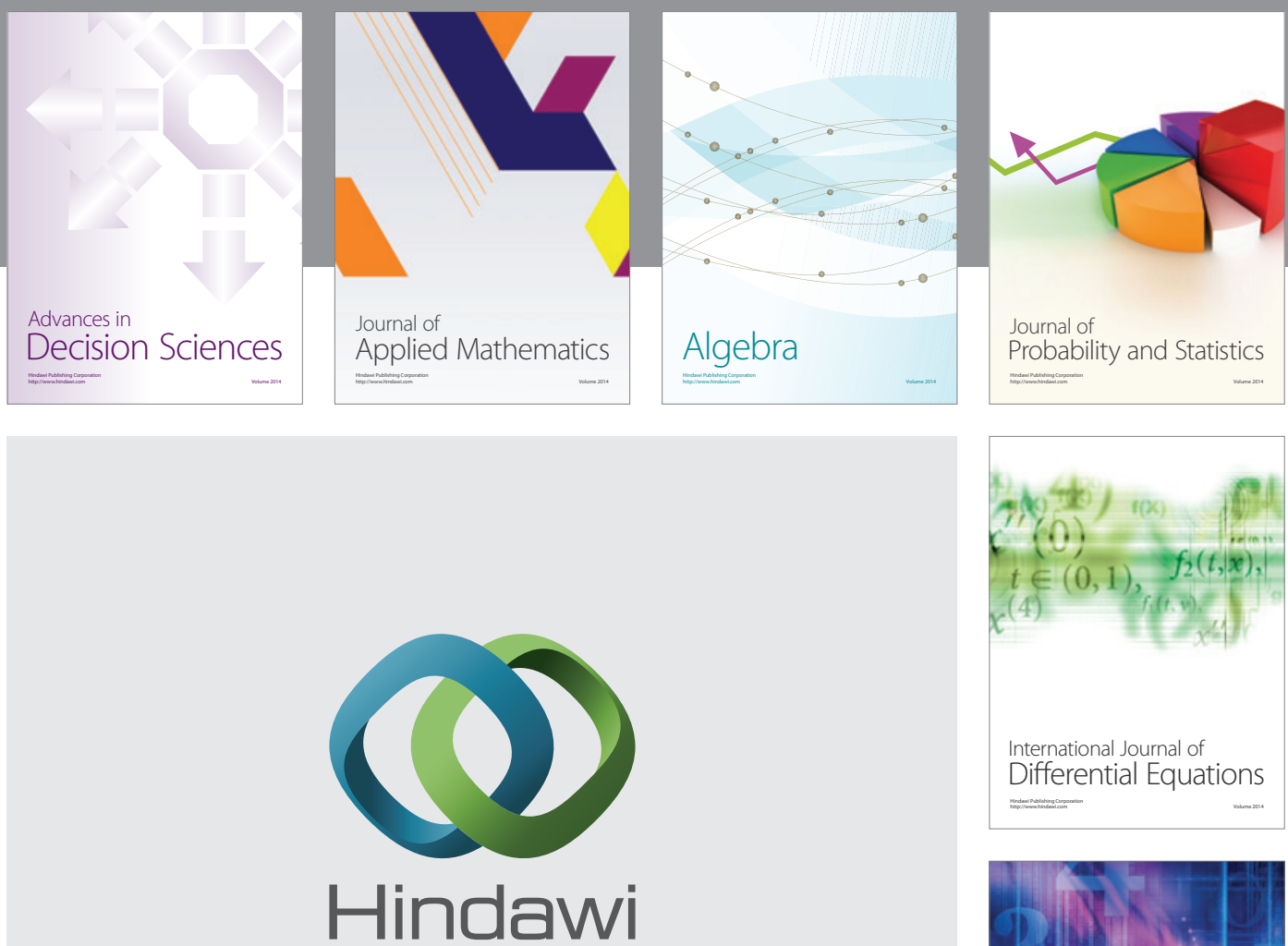

Submit your manuscripts at http://www.hindawi.com
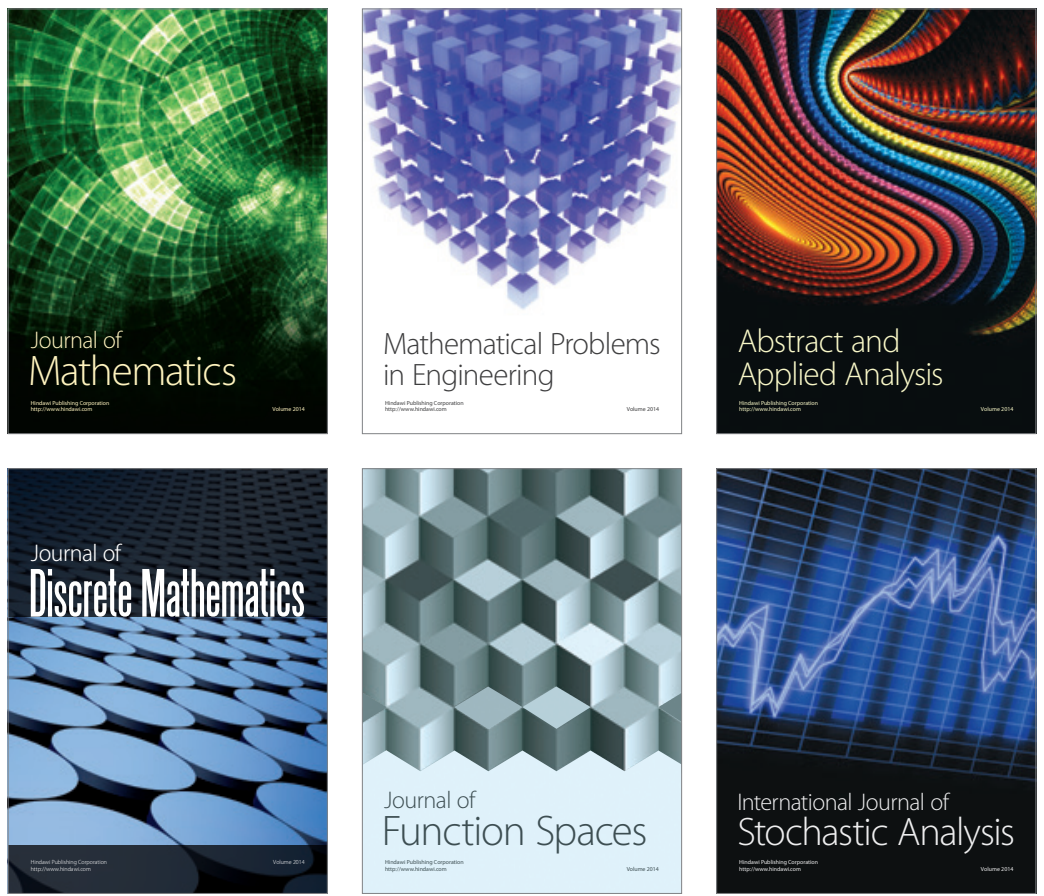

Journal of

Function Spaces

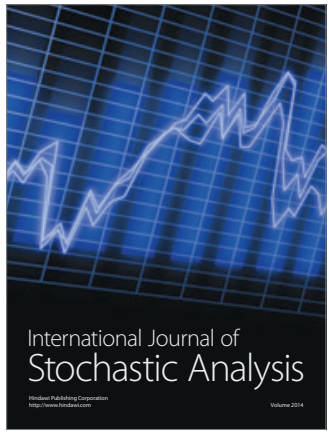

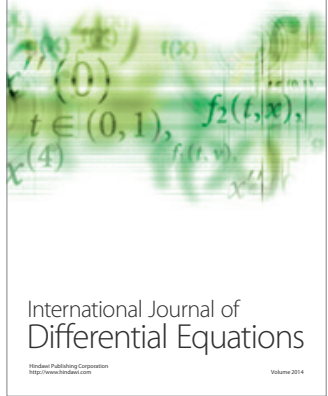
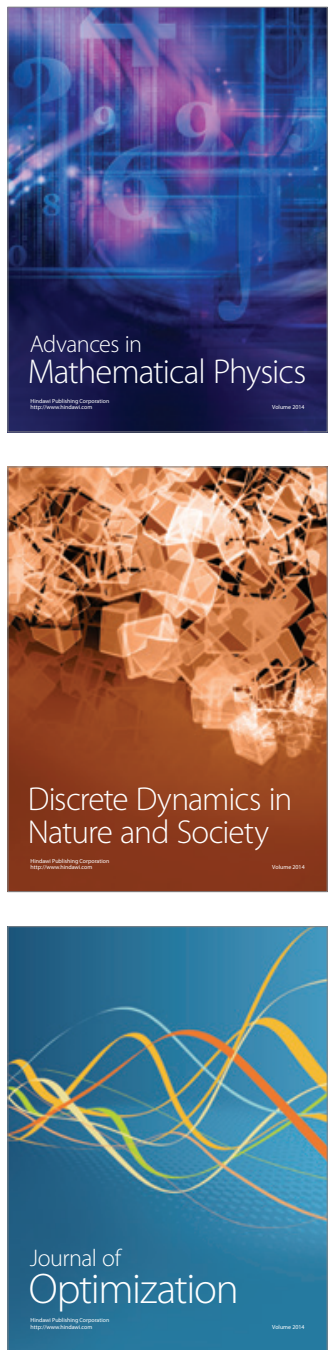